\title{
Is Mycoplasma pneumonia a potential factor for hemoptysis related to pulmonary vascular malformations? A retrospective cohort study of 27 children
}

\section{Hongyu Kuang}

Chongqing Medical University Affiliated Children's Hospital

\section{Qiang Li}

Chongqing Medical University Affiliated Children's Hospital

\section{Qijian Yi}

Chongqing Medical University Affiliated Children's Hospital

Tiewei Lu ( $\square$ ltw200145@163.com)

Children's Hospital of Chonqing Medical University https://orcid.org/0000-0002-0953-6552

\section{Research article}

Keywords: Hemoptysis; Children; Pulmonary vascular malformations; Mycoplasma pneumonia.

Posted Date: December 2nd, 2019

DOl: https://doi.org/10.21203/rs.2.18025/v1

License: (c) (i) This work is licensed under a Creative Commons Attribution 4.0 International License.

Read Full License 


\section{Abstract}

Background: To summarize characteristics of hemoptysis in children associated with pulmonary vascular malformations, and to identify the pathological mechanism, potentially. Methods: Children were recruited who were diagnosed with hemoptysis for pulmonary vascular malformations without congenital heart disease (CHD). The demographics, history, clinical characteristics, laboratory findings, pathogens testing, imaging outcomes and prognosis were systemically reported. The nonparametric Mann-Whitney U test using SPSS 24.0 was employed to compare the continuous laboratory variables, and Fisher's exact test was applied for data enumeration. A P-value of $<0.05$ was considered statistically significant. Results: In sum, 27 children were enrolled in this retrospective study with a medium age of 9.1 years old, among whom $9.1 \%$ were in preschool-group, $72.7 \%$ in school-group, and $18.2 \%$ in adolescent-group. Different hemoptysis degrees were observed from individuals, and the moderate and severe conditions were commonly in elder children. Meanwhile some antecedent symptoms were recorded, mainly cough and fever before hemoptysis occurrence. Pathogens testing indicated proofs for infection of MP in $68.2 \%$ patients, and $37.5 \%$ were further identified with MP-DNA-PCR. Images revealed interstitial changes in most hemoptysis cases, especially in the inferior lobe of right lung for cases with MP infection, and endovascular contrast detected 24 patients with abnormal bronchial arteries (BAs) causing bronchial artery-pulmonary artery fistula and 3 patients suffered from pulmonary arteriovenous fistula (PAVF). After the transcatheter plug closure, a recurrent hemoptysis occurred in 8 patients suffered from an infection of MP initially, and the OR of clinical worsening in Group 1 where cases were considered with an infection of MP, was 2:1. Conclusions: Pulmonary vascular malformations can be commonly detected in elder children. An infection of MP possibly acts as a potential trigger inducing the rupture of affected vessels, which could take a higher risk of clinical worsening.

\section{Background}

Hemoptysis is defined as the expectoration of blood from the lower respiratory tract. As reported, the respiratory infection is the commonest cause in pediatric hemoptysis, almost self-limited ${ }^{[1-2]}$. For a massive blood loss, however, it could be a life-threatening condition requiring approaches for a stabilization of airways. Those approaches included a clear cause, an identification of bleeding sites and

a controlling the continuous hemorrhage, otherwise leading to the acute respiratory distress ${ }^{[3]}$. Pulmonary vascular malformation is illustrated as a cryptogenic cause, accounting for $6 \% \sim 40 \%$, which leads to a massive hemoptysis ${ }^{[4-7]}$. The vascular malformation mainly includes abnormal BAs, PAVFs and collateral arteries in recent studies, and it should be distinguished from other etiologies, such as bronchiectasis, pulmonary tuberculosis and idiopathic pulmonary hemosiderosis (IPH). There exists an unclear pathological mechanism in pediatric hemoptysis with aberrant vessels, while a remodeling of arteries and a compromised pulmonary ventilation could be observed then ${ }^{[8-9]}$. Recently, it is considered that a poor ventilation, hypoxia and an increased blood perfusion could accelerate the process of vascular anomaly where a respiratory infection could act as an important factor ${ }^{[10-11]}$. Previous studies have stated rare proofs about pathogens in hemoptysis, especially associated with pulmonary vascular malformations. 
Consequently, this study was conducted in order to describe characteristics of hemoptysis resulted from different types of pulmonary vascular malformations in children for an early diagnosis and treatment, in addition to potential factors leading to clinical worsening, including recurrence and death. At the same time, a possible pathological mechanism should be probed into.

\section{Methods}

From a technology import to Jan. 2018 in our center, all patients complained of hemoptysis were recorded, and only those who were diagnosed with pulmonary vascular malformations (aberrant BAs, PAVFs and collateral arteries) could be recruited eventually. These patients were with a medium age of 9.1 years old (ranging from 4.2 to 16.0 years old). The diagnosis of hemoptysis in children was carried out according to the standard published in China in 2014. The male to female ratio of vascular malformations in angiographic graphs was about 1:2. According to the patients' age, each patient was classified into pre-school age group ( $<6$ years), school age group (6 12 years) or adolescents group (12 18years). And those patients who displayed any of the following characteristics were excluded from this study: 1) associated with CHD; 2) any evidence indicating other causes of hemoptysis, such as bronchiectasis, tuberculosis infection, IPH, etc.; 3) with unclear etiology. As mentioned ${ }^{[12]}$, we have followed a standard assessing hemoptysis severity as mild, moderate and severe according to the blood loss per 24 hours. And in order to lessen the bias caused by body weight, 3 specific body weight categories of blood loss per 24 hours per mean body weight (MBW) were specified in our observation. The cause was determined with a combination of thorough history, laboratory tests, anamnestic details and non-invasive chest images, and invasive procedures were conducted when available. Some clinical symptoms associated with hemoptysis occurrence in respiratory (fever, cough), gastrointestinal (nausea, vomiting and abdominal pain) and circulatory systems (palpitation, fatigue, anhelation and pale) were observed. The time interval between the antecedent manifestations and hemoptysis event has been computed.

In consideration of infectious causes, an imperative workup was composed of tests for bacteria. Particleassay was conducted for specific antibodies for $M P$, with a proof of recent infection of MP during a higher titer ( $\geq 1: 160)$. MP-DNA-PCR in respiratory secretions has the advantages of high accuracy, sensitivity and specificity, which dynamically changed with the course of the disease, and pulmonary function test (PFT) was performed to evaluate the condition of pulmonary ventilation. Chest image, including chest X-ray and computed tomography (CT), play an important role in identifying pulmonary disorders and infiltrative changes. In addition, endovascular contrast differentiated pulmonary solid masses with vascular malformations and bronchoscopy excludes the possibility of foreign body in the trachea and a rough view of bleeding site. Finally, digital subtraction angiography (DSA) was carried out as the golden criteria, including aorto-arteriography, pulmonary arteriography and a selective trace of the

originations of target vessels ${ }^{[13-15]}$. Laboratory outcomes, pulmonary ventilation, diagnostic fields, and count of abnormal arteries were compared between the MP group (Group 1) and the non-MP group (Group 2). A standard pharmacotherapy for hemoptysis has been undertaken in each case, and once a specific diagnosis of vascular malformations, transcatheter plug occlusion is instantly recommended with a micro 
vascular plug ${ }^{[16-17]}$. Surgical management, such as ligation of vessels or lung resection, is as alternative choice once interventional one failed to control hemorrhage. And the objectives of the ongoing prognosis were followed up regularly, observing and recording the postoperative symptomatic hemoptysis and angiographic outcomes. Additionally, all procedures above could be conducted for these participants with a consent from parents.

All continuous outcome data were expressed as mean \pm standard difference (SD). The nonparametric Mann-Whitney $U$ test was used to compare the continuous laboratory variables between the groups (SPSS 24.0). Fisher's exact test was applied for data enumeration. A $P$-value of $<0.05$ was considered to be statistical significant. The odds ratio (OR) of clinical worsening, including recurrence and death, was calculated to evaluate the potential factors.

\section{Results}

In sum, twenty-seven patients were registered for hemoptysis with pulmonary vascular malformations where $88.9 \%$ of patients were with abnormal BAs and $11.1 \%$ were with PAVFs. The age distribution was recorded as 2 patients were in preschool-group, and 20 patients were in school-group in addition to 5 patients in adolescent-group. Meanwhile, it also demonstrated that the distribution of hemoptysis degree affected by age that almost all the moderate and severe conditions were observed in elder children, including $76.2 \%$ in school-group and $23.8 \%$ in adolescent-group. Additionally, findings indicated that hemoptysis was with a presence of moderate to massive condition when related to abnormal BAs, mostly. When associated with PAVFs, however, symptomatic hemoptysis was unobvious with little blood in sputum or vomitus. At the initial point of hemoptysis, associated with which the common symptoms with the highest frequency were cough $(74.1 \%)$, fever (29.6\%), abdominal pain $(14.8 \%)$, vomit $(7.4 \%)$ and circulation manifestations (22.2\%). And the mean interval duration between the antecedent symptoms and hemoptysis was calculated as $3.9 \pm 3.0$ days. In these series, pathogen test revealed no specific bacteria except for MP. Serum level of MP-Ab were tested in 18 individuals showing a positive rate of 83.3\%. And it indicated a level of $\geq 1: 320$ titers in 12 children and a level of $\geq 1: 160$ titers in 3 children where these 15 children were diagnosed with a current $M P$ infection according to the diagnostic criteria and rolled in Group 1, eventually. Furthermore, MP-DNA-PCR identified 37.5\% patients were detected as a high copy in Group 1. The other 12 patients without proofs for MP infection were defined as Group 2. The demographic characteristics between groups were recorded in Table 1. It showed there existed no obvious difference in distribution of age $(P=.439)$ and gender $(P=.420)$. However, in terms of hemoptysis degree, we found that most severe conditions of massive hemoptysis existed in patients suffered from MP infection at that point, showing a statistical significance $(P=.008)$. Blood routine examinations were conducted in each individual, presenting similar outcomes between groups in WBC count $(P=.733)$, in neutrophil accounting $(P=.107)$, and in hemoglobin level $(P=.884)$. Meanwhile, we found that five episodes, about $62.5 \%$, were observed an abnormal FVC or V25-V75. And it showed three patients from Group 1 with a significant decrease of pulmonary ventilation function, who manifested a massive hemoptysis. 
Graphic findings indicated diagnostic yields. As to CT, this procedure helped a $30 \%$ higher detectable rate of pulmonary lesions than initial radiographs. Currently, a sum of 25 children were detected with diagnostic fields where most of locations were presented in the right lung, especially in Group $1(P=.006)$. All patients in patients with $M P$ infection were detected with definitive lesions, including glassy degeneration $(20.0 \%)$, flocculent shadows $(20.0 \%)$, inflammatory infiltration $(20.0 \%)$, pulmonary atelectasis (13.3\%), consolidation (13.3\%), diffuse patchy shadow (6.7\%) and bronchial arterial enlargement (6.7\%). Furthermore, the distribution of lesions was focused on the right inferior lobe. Totally, 41 detectable anomalous BAs were found in 24 children, and single or multiple PAVFs were detected in 3 children by angiography. As golden criteria of pulmonary vascular malformations, it hinted smoking phenomenon locating at the sites of vascular ruptures and bleeding. About $50 \%$ of patients had more than two abnormal bronchial vessels corresponding to each other. These abnormal arteries were shown to originate from different sites of systemic circulation, which connected and jointly delivered systemic-topulmonary blood perfusion. Twenty-six, about $63.4 \%$, tortuous or hypertrophy arteries were from T5-T6 level of thoracic aorta, along the bronchial tree entering the pulmonary fields. Eight BAs (19.5\%) were from descending aorta beyond T5-T6 level, and two BAs (4.9\%) were involved in the aortic arch, four BAs (9.8\%) were the branches of subclavian arteries in four independent cases. One abnormal artery was considered from the hepatic aorta. As to patients with MP infection in Group1, 14 patients were with abnormal BAs, as shown in Fig.1. Meanwhile, the counts of abnormal arteries were different with or without MPinfection $(P=.026)$.

Twenty-three patients were treated transcatheter plug occlusion, including 20 patients with abnormal BAs and 3 patients with PAVFs. In Group 1, 14 patients received interventional therapy and one patient received the lung resection for an aberrant artery originating from the hepatic artery. In Group 2, two patients chose traditional medicine (antibiotics and hemostatic drugs) for a mild hemoptysis and one patient underwent a ligation of the abnormal BA from right subclavian artery using a thoracoscopic guidance. The others were undertaken a treatment of transcatheter plug occlusion. After the transcatheter plug occlusion, findings showed an obvious reduction of contrast medium shunts according to an instant angiogram, indicating an effective procedure, while there existed 14 recurrence episodes in 8 patients with abnormal BAs in Group1 $(P=.002)$. Additionally, all of cases with recurrent hemoptysis were categorized as a massive hemoptysis, with a titer of $M P-A b \geq 1: 320$ in serum, primarily. Furthermore, a lasting high level of MP-Ab was observed in a recurrent state, and details of recurrent cases were shown in Table 2. One patient received the lung resection died after surgery for one week. The OR of clinical worsening in patients with MPinfection was about 2:1. Moreover, the even-free survival rate of patients was portrayed in Fig.2.

\section{Discussion}

Hemoptysis is a significant clinical entity seen in pediatric patients ${ }^{[1,19]}$. It is proved that hemorrhage caused by pulmonary vascular malformations mostly occurs due to the rupture of distal anastomoses. And bronchial circulation possesses a remarkable capacity for remodeling, presenting as angiogenic and enlargement factor, thus leading to BPFs. Additionally, infection is considered as a common cause of 
enlargement of bronchial circulation, successively increasing the blood flow, thus increasing the risk in a rupture of aberrant vessels. Currently, our study proved that hemoptysis caused by vascular malformations were perhaps highly correlated with MPinfection. As is known, MPinfection that was commonly considered to be prevalent in children between 5 to 15 years old ${ }^{[20]}$. And we found the pediatric patients complained of hemoptysis with aberrant vessels were with a medium age of 9.06 years old, ranging from 4.2 to 16.0 years old. Additionally, in our study, various clinical manifestations and time interval before symptomatic hemoptysis are accordance with MP infection, combined with about $90 \%$ positive rate of MP specific test. It commonly causes interstitial inflammatory changes and are seen during the chest radiological examinations, commonly damaging the right lung and locating in the inferior lobe ${ }^{[21]}$. The rupture location of vasculature of hemoptysis in cases was more concentrated in the middle to inferior lobe in the right side of pulmonary region. These results were in contrast with the chest images of $M P$ infection, and it was in accordance with the imaging findings. It was concluded that $M P$ invasion was probably involved in the pediatric hemoptysis with vascular malformations. Additionally, it was possible that the MP actively presented in the acute phase for outcomes of MP-PA and MP-PCR. It can damage endothelium, activate immune systems and induce vascular enlargement and sharply increase the blood flow. Finally, MP infection was one of the most important triggering factors for vascular rupture of anastomosis in hemoptysis subjects ${ }^{[22-23]}$. Both large and small airway functions can be damaged with $M P$ infection during the acute phase ${ }^{[24-25]}$. It is one of the smallest known free-living, self-replicating bacteria. The pathogenesis of $M P$ infection involving complex mechanisms, mainly the direct damage mechanism, membrane fusion damage and immune damage were reviewed. The adhesion of MP onto the alveolar macrophages, alveolar epithelial, vascular epithelia, fibroblasts etc. can interfere with the structural lesions ${ }^{[26]}$. Meanwhile, $M P$ is considered to be invasive and can induce extensive vascular degeneration. Inflammatory reaction in the airways can lead to inflammatory damage during $M P$-induced inflammatory reaction. Studies indicated that in children with $M P$ infection, the serum level of interleukins, intercellular adhesion molecule- 1 and tumor necrosis factor are markedly increased. These were involved the inflammation and infiltration of the affected tissues, and may lead to increased bronchial reaction, aggravation of vascular damage and atherosclerosis ${ }^{[27]}$. So, for an intravascular movement with blood flow and colonization, MP can damage the wall of segmental vessels by injuring the vascular endothelial cells, intercellular tight junction and fibroblasts. Bronchial circulation contributed to the recruitment of inflammatory cells by triggering the immune function to the mucosa of airways. This can be triggered to improve the volume from BAs to the affected vessels ${ }^{[28]}$. A lasting blood perfusion can cause changes in the vessel dilation, especially in the "bronchopulmonary anastomosis", which could be considered as a located aneurysms or pseudo-aneurysms secondary to MPinfection.

Pulmonary bloodstreams supplied by dual circulation systems, which include bronchial circulation for delivering oxygenated blood to the walls of conducting airways and pulmonary vessels, and pulmonary circulation for pulmonary ventilation. The bronchial vasculature plays an important role in health and diseases, especially in hemoptysis, approximately $90 \%{ }^{[29]}$. Also, branching of the intrapulmonary arteries along the length of the bronchial tree down to the terminal bronchioles forms a vast network of capillaries. Different from CHD with aortopulmonary arteries, the bronchial circulation or pulmonary 
circulation of these hemoptysis individuals may also remodel by triggering some unknown factors during the embryonic period ${ }^{[30]}$. Maternal hypoxia may contribute to oxygen deficiency of pulmonary tissues, combining with an unestablished ventilation function. Low oxygen can promote the regulation of angiogenic gene expression, such as vascular endothelial growth factor (VEGF) in triggering angiogenesis. This possibly causes anomalous systemic arteries to pulmonary region ${ }^{[31]}$. Aberrant bronchial circulation accounted a lot in our survey according to the imaging description [32] Physiologically, the right lung contains several vascular branches for supply, cluing for high oxygen demands. The dilated changes in the bronchial artery anatomy are more likely to increase the perfusion heterogeneity for oxygen provision, with successive branches in the vascular tree in the right inferior lobe.

The bronchial circulation from systemic circulation showed high-resistance and low-capacitance, while the pulmonary arteries allowed the identification of low-resistance circulation, which can lead to a persistent dilation in BAs ${ }^{[33]}$. Our understanding regarding the findings showed some bloodstreams from anomalous systemic arteries still flow to the pulmonary arteries consistently during the postnatal development. Also, the distal microvasculature forms multiple anastomoses and was referred to as "bronchopulmonary anastomotic". Persistent bloodstreams lead to an increased pressure in these anastomoses, like some cystic changes, particularly in the pulmonary capillary sections ${ }^{[34]}$. There exist a mismatch of volume and perfusion in the pulmonary region in a condition with $M P$ infection. The decreasing level of FVC V25 V75 indicated dysfunction of small airways in some cases who underwent PFT in our study. Gas exchange can only occur if there was a tight match between ventilation and perfusion at the level of the alveolar capillaries and it indicates a tight coupling of ventilation and perfusion results in minimal homogeneity of ventilation/perfusion ratio (V/Q) ${ }^{[35]}$. Rahn et al. demonstrated that $\mathrm{V} / \mathrm{Q}$ were significantly higher in the upper lobes of the lungs in animal trials ${ }^{[36]}$. Kaneko and coworkers ${ }^{[37]}$ have supported the opinion that gravity was responsible for the vertical gradient in $\mathrm{V} / \mathrm{Q}$ ratios of inferior lobes. For hemoptysis with respiratory infection, the $\mathrm{V} / \mathrm{Q}$ ratio demonstrated an obvious imbalance. Structural changes along their occurrence in alveolar tissues reduce the number of functional alveoli progressively. This in turn creates an obvious loss of functional gas exchange units as well as lung compliance and alters the ventilation-perfusion relationships causing restrictive ventilator disorders. As discussed above, it is very likely that a compromised ventilation in hemoptysis cases lead to hypoxia further. Then the compensatory arterial blood flow from the BAs with higher oxygen content to supply the pulmonary tissues in a short time ${ }^{[38]}$. This scenario also can provide an increasing pressure of located vessels for regulation, especially in the capillaries. Meanwhile, one of the commonest manifestation of $M P$ is a rough cough for lesions of respiratory ciliary system. A cough reflex could certainly increase the pressure in the thoracic cavity, especially during a persistent and spastic cough, easily leading to the rupture of small vessels of abnormal BAs or fistulas of pulmonary arteriovenous.

\section{Conclusions}


We concluded that it should not be denied the rupture of abnormal vessels in pulmonary region remain crucial factors for the occurrence of pediatric hemoptysis, such as abnormal BAs and PAVFs. Pediatric hemoptysis is concentrated in elderly children with respiratory infection, especially with $M P$ infection which could induce a weak recovery $(\mathrm{OR}=2: 1)$. Therefore, an early diagnosis of children with hemoptysis, with a combination of clinical manifestations in addition to accessory examinations, is required. More emphasis should be placed on the etiological therapy as well as suitable treatment for vascular malformations that trigger hemoptysis. So, the possible mechanisms for pediatric hemoptysis related to pulmonary vascular malformations with MPinfection were summarized in Fig. $\mathbf{3}$.

Some shortcomings still existed in our study: 1) The outcomes of this study were obtained from a singlecenter with a relatively small sample size; 2) Additionally, there exists a selection bias in data collection and classification of hemoptysis severity; 3 ) All data were retrospective in a hemoptysis cohort with vascular malformations; 4) No controlled group existed. Hence, to confirm the above findings, a multicenter, larger sample size and randomized controlled trials should be conducted.

\section{Declarations}

\section{Ethics approval and consent to participate}

All tests, medications and procedures performed in the enrolled patients in our center were in accordance with the ethical standards of Children's Hospital of Chongqing Medical University. And informed consent was obtained from parents of all individual participants in the study.

\section{Consent for publication}

Not applicable.

\section{Availability of data and materials}

The datasets were generated during the current study are not publicly available due to a privacy of enrolled participants, but they are available from the corresponding author on reasonable request.

\section{Competing interests}

The authors all declare that they have no competing interests.

\section{Funding}

All authors have declared that no funding existed in this study.

\section{Authors' contributions}

All authors contributed substantially to the study design, data collection, data analyses, and the writing of the manuscript. Hongyu Kuang and Qiang Li conducted the principal investigation, study design, data 
collection, statistical analysis, preparation and writing of this manuscript; Qijian Yi acted as a role contributing to an interventional procedure and proofreading; Tiewei Lu has participated in the study design, evaluation of all outcomes and manuscript. All typographical and grammatical errors could be checked and corrected by authors. And the corresponding author has confirmed all contributing authors gave permissions to be named in this study.

\section{Acknowledgements}

Not applicable.

\section{References}

1. Gaude GS. Hemoptysis in children [J]. Indian Pediatr, 2010; 47(3): 245-254.

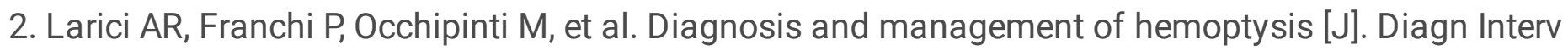
Radiol, 2014; 20(4): 115-118.

3. Godfrey S. Pulmonary hemoptysis in children [J]. Pediatr Pulm, 2004『37(6):476-484.

4. Sidman JD, Wheeler WB, Cabalka AK, et al. Management of acute pulmonary hemorrhage in children[J]. Laryngoscope, 2010, 111(1):33-35.

5. AbuKishk I, Klin B, Eshel G. Hemoptysis in children: a single institutional experience [J]. Pediatr Emerg Care, 2012; 28(11): 1206-1210.

6. Sim J, Kim H, Lee H, et al. Etiology of hemoptysis in children: a single institutional series of 40 cases [J]. Allergy Asthma Immun, 2009; 1(1): 41-44.

7. Nugent Z, Vicente Oliveira MD, lan MacLusky MD, et al. Bronchial artery-pulmonary artery malformation as a cause of cryptogenic hemoptysis [J]. Pediatr Pulmon, 2013, 48(9):930-933.

8. Jiang S, Sun XW, Jie B, et al. Endovascular Embolization of an Aberrant Bronchial Artery Originating from the Vertebral Artery in a Patient with Massive Hemoptysis[J]. Cardiovasc Interv Rad, 2013; 37(4): 1099-1102.

9. Ponnuswamy I, Sankaravadivelu ST, Maduraimuthu P, et al. 64-detector row CT evaluation of bronchial and non-bronchial systemic arteries in life-threatening haemoptysis[J]. Brit J Radiol, 2012, 85(1017):666-672.

10. Yamamura K, Nagata H, Ikeda K, et al. Efficacy of bosentan therapy for segmental pulmonary artery hypertension due to major aortopulmonary collateral arteries in children [J]. Int J Cardiol, 2012, 161(1):1-3.

11. Dokumcu Z, Ozcan C, Alper H, et al. Pulmonary arteriovenous malformation in children [J]. Pediatr Int, 2015; 57(4): 708-711.

12. Yao L, Shen KL. Hemoptysis and bronchial artery-pulmonary artery malformation [J]. China Journal Applied Clinical Pediatrics, 2014; 29(6): 1203-1206.

13. Khalil A, Fartoukh M, Tassart M, et al. Role of MDCT in identification of the bleeding site and the vessels causing hemoptysis [J]. Am J Roentgenol, 2007, 188(2): 117-125. 
14. Ittrich H, Klose H, Adam G. Radiologic Management of Hemoptysis: Diagnostic and Interventional Bronchial Arterial Embolisation [J]. Fortschr Rontgenstr, 2014, 187(4):248-259.

15. Singh D, Bhalla AS, Veedu PT, et al. Imaging evaluation of hemoptysis in children[J]. World J Clinical Pediatr, 2013; 2(4): 54-54.

16. Boudjemline Y. Covidien micro vascular plug in congenital heart diseases and vascular anomalies: A new kid on the block for premature babies and older patients[J]. Catheter Cardio Inter, 2017; 89(1): 114-119.

17. Cordovilla R, Bollode ME, NuñezAres A, et al. Diagnosis and Treatment of Hemoptysis [J]. Archivos De Bronconeumologia, 2016; 52(7): 368-377.

18. Jiang ZF, Shen KL, Shen Y. Zhu Futang Practice of Pediatrics (8th Edition) [M]. Ren Min Wei Sheng Chu Ban She, 2015: 1280-1283.

19. Batra PS, Holinger LD. Etiology and management of pediatric hemoptysis[J]. Arch Otolaryngol, 2001; 127(4): 377-382.

20. Medjo B, Atanaskovicmarkovic M, Radic S, et al. Mycoplasma pneumonia as a causative agent of community-acquired pneumonia in children: clinical features and laboratory diagnosis[J]. Ital $\mathrm{J}$ Pediatr, 2014, 40(1): 104-107.

21. Kicinski P, Wisniewska-Ligier M, Wozniakowska-Gesicka T. Pneumonia caused by Mycoplasma pneumoniae, and Chlamydophilapneumoniae, in children-comparative analysis of clinical picture[J]. Adv Med Sci, 2011, 56(1): 56-63.

22. Williamson J, Marmion BP, Worswick DA, et al. Laboratory diagnosis of Mycoplasma pneumoniae infection 4. Antigen capture and PCR-gene amplification for detection of the Mycoplasma: Problems of clinical correlation [J]. Epidemiol Infect, 1992, 109: 519-537.

23. Yoo SJ, Oh HJ, Shin BM. Evaluation of four commercial IgG-and IgM-specific enzyme immunoassays for detecting Mycoplasma pneumoniae antibody: Comparison with particle agglutination assay [J]. J Korean Med Sci, 2007, 22(5): 795-801.

24. Wu LX, Wu M, Gu DP. Pulmonary functions in children with segmental Mycoplasma pneumonia pneumonia [J]. Chinese Journal of Contemporary Pediatrics, 2011, 13(3):185-187.

25. Kannan TR, Musatovova O, Balasubramanian S, et al. Mycoplasma pneumoniae Community Acquired Respiratory Distress Syndrome toxin expression reveals growth phase and infection [J]. Mol Microbiol, 2010,76(5): 1127-1141.

26. He J, Liu M, Ye Z, et al. Insights into the pathogenesis of Mycoplasma pneumoniae[J]. Mol Med Rep, 2016, 14(5): 4030-4036.

27. Lee KE, Kim KW, Hong JY, et al. Modulation of IL-8 Boosted by Mycoplasma pneumoniae, lysate in Human Airway Epithelial Cells [J]. J Clin Immunol, 2013, 33(6): 1117-1125.

28. Barman SA, Pauly JR, Isales CM. Canine pulmonary vasoreactivity to serotonin: role of protein kinase C and tyrosine kinase [J]. Am J Physiol, 1997; 272(2): 740-747.

29. Suresh K, Shimoda LA. Lung circulation [J]. Compre Physiol, 2016, 6(2): 897-943. 
30. Warren RL, Powell WJ Jr. Acute alveolar hypoxia increases bronchopulmonary shunt flow in the dog [J]. J Clin Invest, 1986, 77(5): 1515-1524.

31. Srinivasan S, Chitalia V, Meyer RD, et al. Hypoxia-induced expression of phosducin-like 3 regulates expression of VEGFR-2 and promotes angiogenesis[J]. Angiogenesis, 2015,18(4): 449-462.

32. Suzuki K, Kamata N, Tanaka J, et al. Clinical investigation of the branching formation of the bronchial arteries in the Japanese[J]. Nihon Igaku Hoshasen Gakkai Zasshi Nippon Acta Radiologica, 1989, 49(8): 979-985.

33. Osaki $\mathrm{S}$, Nakanishi $\mathrm{Y}$, Wataya $\mathrm{H}$, et al. Prognosis of bronchial artery embolization in the management of hemoptysis [J]. Respiration, 2000, 67(4): 412-416.

34. Murphy PA, Hynes RO. Alternative splicing of endothelial fibronectin is induced by disturbed hemodynamics and protects against hemorrhage of the vessel wall[J]. Arterioscl Throm Vas, 2014, 34(9): 2042-2050.

35. Glenny RW, Thomas RH. Spatial Distribution of Ventilation and Perfusion: Mechanisms and Regulation[J]. Compr Physiol, 2011, 1(1): 375-375.

36. Rahn H, Sadoul P, Farhi L, et al. Distribution of ventilation and perfusion in the lobes of the dog's lung in the supine and erect position. J Appl Physiol, 1956, 8(4): 417-426.

37. Kaneko K, Milic-Emili J, Dolovich MB, et al. Regional distribution of ventilation and perfusion as a function of body position [J]. J Appl Physiol, 1966, 21(3): 767-777.

38. Mcdonald D. Angiogenesis and remodeling of airway vasculature in chronic inflammation[J]. Am J Resp Crit Care, 2001, 164(2): 39-45.

\section{Tables}

Table1 Comparative outcomes between hemoptysis groups

\begin{tabular}{|c|c|c|c|}
\hline Column & Group $1(\mathrm{n}=15)$ & Group $2(\mathrm{n}=12)$ & $P$-value \\
\hline Age $($ mean $\pm \mathrm{sd})$ & $9.5 \pm 2.0$ & $8.6 \pm 3.8$ & .439 \\
\hline Gender (Female) & 9 & 9 & .420 \\
\hline Massive hemoptysis & 10 & 2 & .008 \\
\hline WBC $\operatorname{count}\left(\wedge 10^{9} / \mathrm{L}\right)$ & $9.3 \pm 3.1$ & $9.1 \pm 2.5$ & .733 \\
\hline NEUT (\%) & $71.7 \pm 12.1$ & $60.6 \pm 18.3$ & 107 \\
\hline $\mathrm{Hb}(\mathrm{g} / \mathrm{L})$ & $114.5 \pm 17.4$ & $123.9 \pm 55.6$ & .884 \\
\hline Lesion location*(n) & 11 & 3 & .006 \\
\hline Count of abnormal arteries $(\mathrm{n}) \neq$ & 2.0 & 1.3 & .026 \\
\hline Recurrence (n) & $6 \dagger$ & $0 \dagger \dagger$ & .002 \\
\hline
\end{tabular}


Group1= Cases with $M P$ infection (clinical manifestation combined with chest images and $M P$ test $(+)$ ). Group $2=$ Cases without proofs for $M P$ infection.

* Lesions located in the inferior lobe of right lung; $\ddagger$ A location of pulmonary arteriovenous fistula is counted as one abnormal artery; †One case received the lung resection; †† Two patients selected the traditional therapy and 1 patient was undertaken a vascular ligation.

Table2. Details of patients with recurrent episodes

\begin{tabular}{|c|c|c|c|c|c|c|c|c|c|}
\hline ies & $\begin{array}{l}\text { Age }^{*} \\
\text { (yrs) }\end{array}$ & Type $^{\dagger}$ & Procedure & $\begin{array}{l}\text { HS } \\
\text { (day) }\end{array}$ & $\begin{array}{l}\text { MP-Ab } \\
\text { at } \\
\text { baseline } \\
\text { (titers) }\end{array}$ & Times $^{\ddagger}$ & $\begin{array}{l}\text { Duration }^{\ell} \\
\text { (mths) }\end{array}$ & $\begin{array}{l}\text { MP-Ab at } \\
\text { recurrent } \\
\text { state(titers) }\end{array}$ & $\begin{array}{l}\text { MP-PCR at } \\
\text { recurrent } \\
\text { state } \\
\text { (copies } / \mathrm{ml} \text { ) }\end{array}$ \\
\hline $\mathrm{e}$ & 9.7 & $\begin{array}{l}\text { Abnormal } \\
\text { BAs }\end{array}$ & TPO & 11 & $1: 320$ & 3 & $\begin{array}{l}11.3 \\
(6.6)\end{array}$ & $>1: 320^{1}$ & - \\
\hline e & 8.8 & $\begin{array}{l}\text { Abnormal } \\
\text { BAs }\end{array}$ & TPO & 15 & $1: 320$ & 3 & $\begin{array}{l}14.0 \\
(3.6)\end{array}$ & $1: 320^{2}$ & $5.85^{\wedge} 10^{5}$ \\
\hline e & 8.4 & $\begin{array}{l}\text { Abnormal } \\
\text { BAs }\end{array}$ & TPO & 14 & $1: 320$ & 3 & $\begin{array}{l}11.0 \\
(5.7)\end{array}$ & $1: 320^{2}$ & - \\
\hline e & 10.0 & $\begin{array}{l}\text { Abnormal } \\
\text { BAs }\end{array}$ & TPO & 13 & $1: 160$ & 1 & $10.0(-)$ & $\begin{array}{l}1: 160^{1} \\
1: 320^{1}\end{array}$ & - \\
\hline e & 9.6 & $\begin{array}{l}\text { Abnormal } \\
\text { BAs }\end{array}$ & TPO & 14 & $1: 160$ & 1 & $9.0(-)$ & $1: 320^{1}$ & - \\
\hline e & 8.1 & $\begin{array}{l}\text { Abnormal } \\
\text { BAs }\end{array}$ & TPO & 8 & $1: 320$ & 1 & $5.3(-)$ & $1: 160^{1}$ & - \\
\hline e & 10.0 & $\begin{array}{l}\text { Abnormal } \\
\text { BAs }\end{array}$ & TPO & 9 & $1: 320$ & 1 & $4.0(-)$ & $1: 320^{1}$ & $2.28^{\wedge} 10^{4}$ \\
\hline e & 8.0 & $\begin{array}{l}\text { Abnormal } \\
\text { BAs }\end{array}$ & TPO & 12 & $1: 320$ & 1 & $1.0(-)$ & - & - \\
\hline
\end{tabular}

*The age of cases with hemoptysis on-set. ${ }^{\dagger}$ Type of pulmonary vascular malformations. ${ }^{\ddagger}$ Times of hemoptysis recurrence. ${ }^{€}$ Interval time from every occlusion to recurrence point. " $M P$-Ab level in serum tested during a recurrent state $(1=$ it was tested in one episode of recurrence; $2=$ it was tested in two episodes of recurrence).

$\mathrm{BA}=$ bronchial artery; $\mathrm{yrs}=\mathrm{years} ; \mathrm{TPO}=$ transcatheter plug occlusion; $M P=$ mycoplasma pneumonia; $\mathrm{Ab}=\mathrm{antibody}$. $\mathrm{HS}=$ hospital stay at initial point.

\section{Figures}



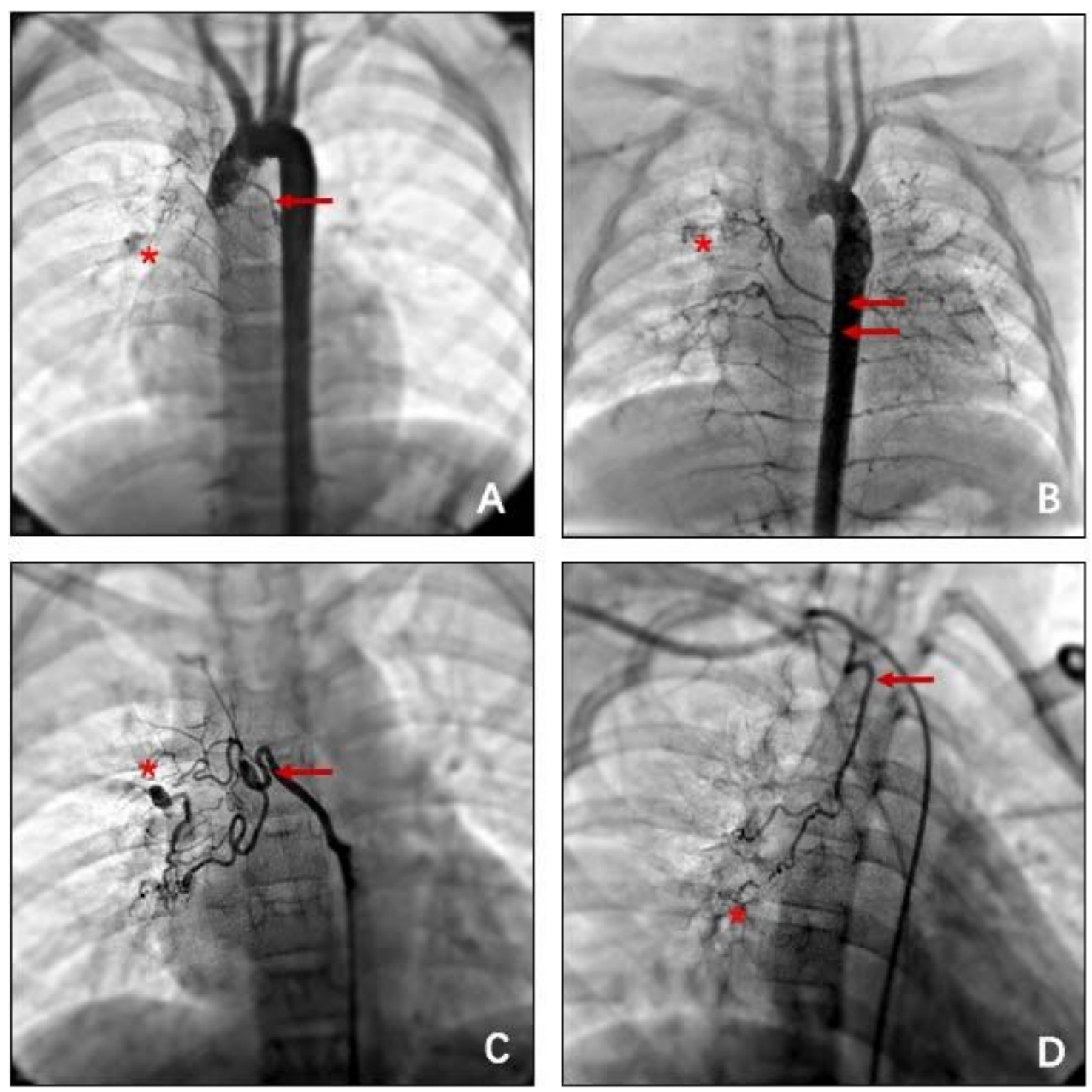

\section{Figure 1}

Abnormal BAs and smoking phenomenon detected by DSA. (arrow: abnormal arteries; asterisk: the site of rupture in vessels) 


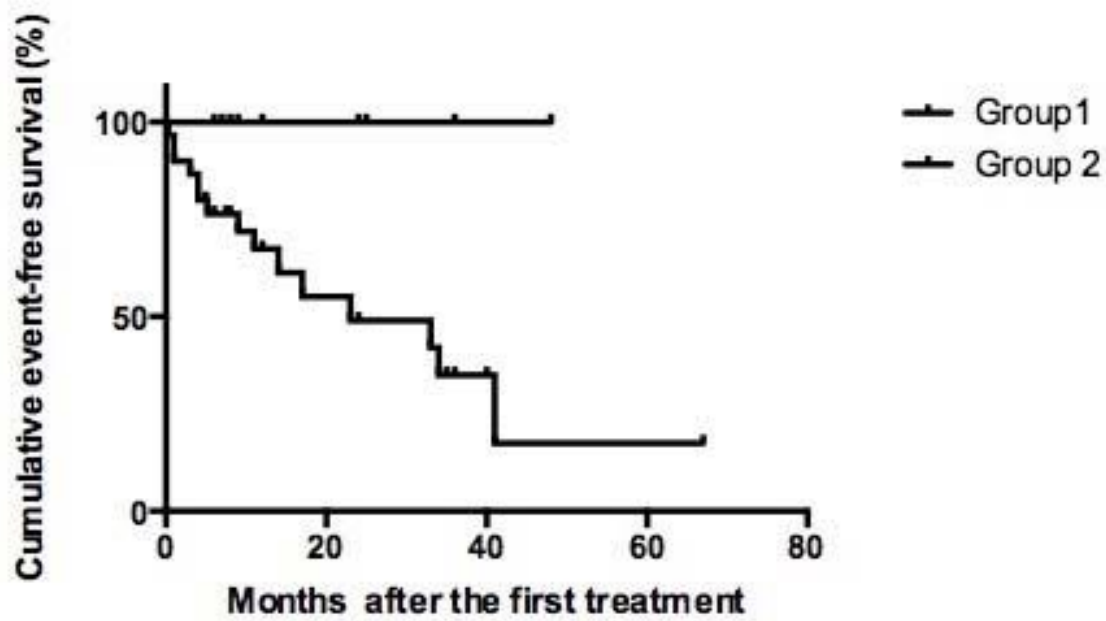

Figure 2

The cumulative event-free survival rate between groups with or without MP infection. 

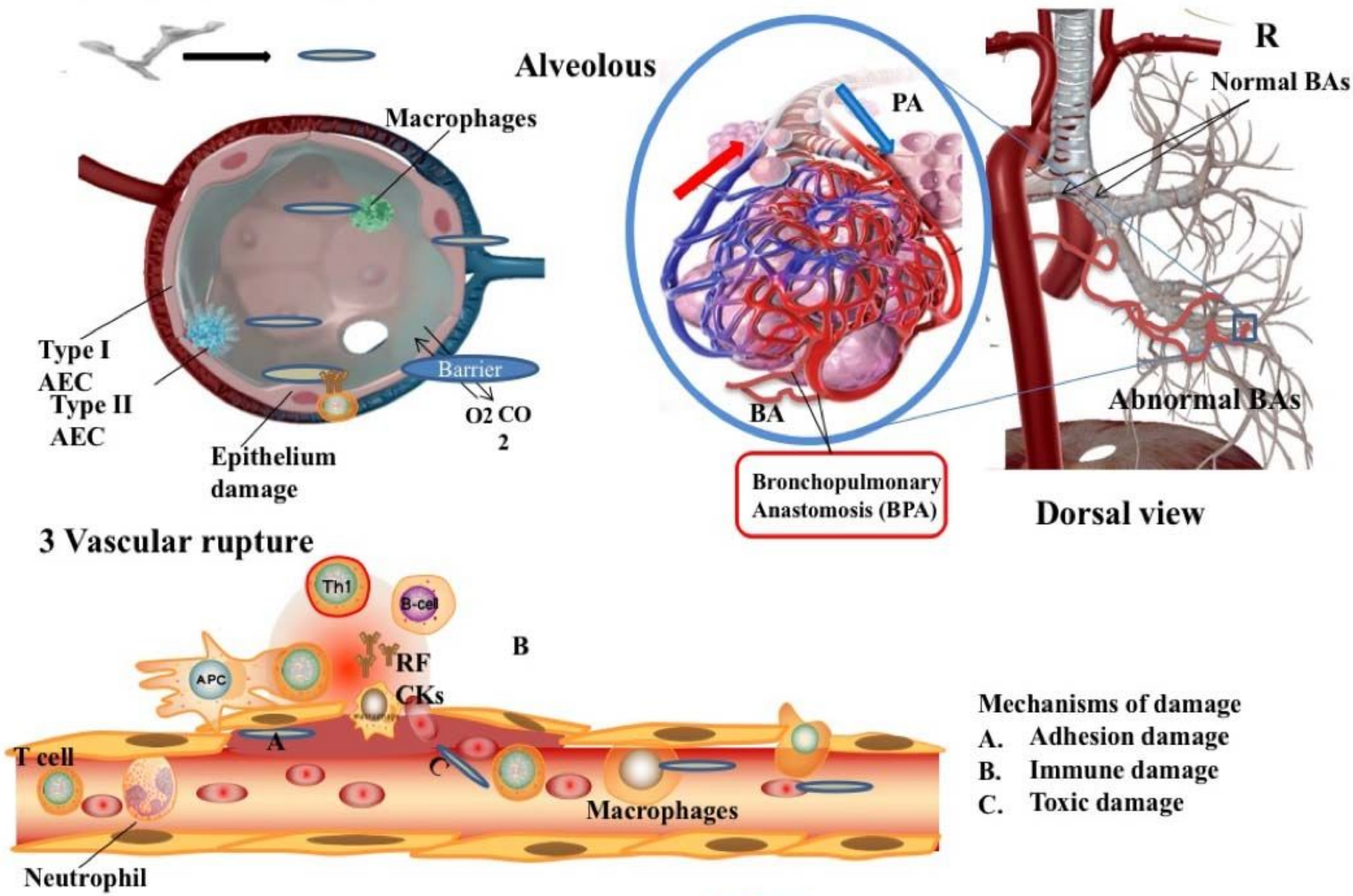

Mechanisms of damage

A. Adhesion damage

B. Immune damage

C. Toxic damage

\section{Neutrophil}

M.Pneumonia $\rightleftharpoons$ Erythrocyte $\odot$ Dilation changes

BAs or BPA

\section{Figure 3}

The possible mechanism of hemoptysis related to pulmonary vascular malformations with MP infection. (And all authors declare that this figure has been portrayed by our own) 